\title{
Stereodynamic control of overlapping resonances in cold molecular collisions
}

\author{
Masato Morita, ${ }^{1}$ Qian Yao $\odot,{ }^{2}$ Changjian Xie,${ }^{2,3}$ Hua Guo, ${ }^{2}$ and Naduvalath Balakrishnan $\oplus^{1, *}$ \\ ${ }^{1}$ Department of Chemistry and Biochemistry, University of Nevada, Las Vegas, Nevada 89154, USA \\ ${ }^{2}$ Department of Chemistry and Chemical Biology, University of New Mexico, Albuquerque, New Mexico 87131, USA \\ ${ }^{3}$ Institute of Modern Physics, Shaanxi Key Laboratory for Theoretical Physics Frontiers, Northwest University, Xian, Shaanxi 710127, China
}

(Received 12 May 2020; accepted 30 June 2020; published 17 July 2020)

\begin{abstract}
Stereodynamic control of resonant molecular collisions has emerged as a new frontier in cold molecule research. Recent experimental studies have focused on weakly interacting molecular systems such as HD collisions with $\mathrm{H}_{2}, \mathrm{D}_{2}$, and He. We report here the possibility of such control in strongly interacting systems taking rotational relaxation in cold collisions of $\mathrm{HCl}$ and $\mathrm{H}_{2}$. Using explicit quantum scattering calculations in full six dimensions it is shown that robust control of the collision dynamics is possible even when multiple (overlapping) shape resonances coexist in a narrow energy range, indicating that cold stereochemistry offers great promise for many molecules beyond simple systems. We demonstrate a striking case where two prominent peaks in overlapping resonances are switched off simultaneously by suitable alignment of the $\mathrm{HCl}$ molecule.
\end{abstract}

DOI: 10.1103/PhysRevResearch.2.032018

Recent progress in cooling and trapping molecules below $1 \mathrm{~K}$ has led to new insights into chemical dynamics, quantum information processing, quantum simulation, and the test of fundamental physics beyond the standard model [1-10]. In particular, the ability to prepare molecules in selected motional, internal, and orientational quantum states has allowed interrogation of molecular events with unprecedented precision. The suppression of higher-order angular momentum partial waves in cold collisions provides an ideal platform to explore state-to-state chemistry with partial wave resolution.

A series of papers by Perreault et al. [11-13] laid the groundwork for cold stereodynamics [14-17] by combining cold molecular beam technology and coherent optical control of molecular states. They demonstrated stereodynamic control of rotational quenching of vibrationally excited HD $(v=$ $1, j=2)$ in cold collisions with $\mathrm{H}_{2}, \mathrm{D}_{2}$, and $\mathrm{He}$ [11-13] in a single molecular beam. The initial molecular state of $\mathrm{HD}$, including the bond axis alignment, was prepared by Stark-induced adiabatic Raman passage (SARP) $[18,19]$. The quenching rate and angular distribution were found to vary significantly depending on whether the HD bond axis is preferentially aligned horizontal (H-SARP) or vertical (V-SARP) to the initial relative velocity vector between the collision partners, providing conclusive evidence of strong stereodynamic effect in $\mathrm{HD}+\mathrm{H}_{2} / \mathrm{D}_{2}$ collisions near $1 \mathrm{~K}$.

While the collision energies involved in the experiment are narrow $(<5 \mathrm{~K})$ and only a limited number of partial waves $(\sim l<5)$ is involved, the experiments do not provide energy or partial wave resolution directly. In a subsequent

\footnotetext{
*Corresponding author: naduvala@unlv.nevada.edu

Published by the American Physical Society under the terms of the Creative Commons Attribution 4.0 International license. Further distribution of this work must maintain attribution to the author(s) and the published article's title, journal citation, and DOI.
}

theoretical study Croft et al. [20] attributed the key feature of the angular distribution of the rotationally deexcited HD to an incoming $d$-wave $(l=2)$ shape resonance centered at about $1 \mathrm{~K}$. The role of isolated (shape) resonances in controlling quenching rate and overall angular distribution of the products was further explored in theoretical studies of $\mathrm{HD}+\mathrm{H}_{2}[21,22]$ and in recent experiments on HD+He [13].

In addition to H-SARP and V-SARP, Perreault et al. [13] illustrated an X-SARP orientation in which the HD bond axis is prepared in a superposition state with axis orientation of $45^{\circ}$ with respect to the collision velocity. Indeed, an explicit stereodynamic control was demonstrated in their experiments as the bond-axis orientation was varied discretely from $0^{\circ}$ to $45^{\circ}$ to $90^{\circ}$ with respect to the collision velocity. This was generalized to an arbitrary orientation of the HD bond axis by Croft and Balakrishnan [21] and Jambrina et al. [22] for $\mathrm{HD}+\mathrm{H}_{2}$, achieving further control of the stereodynamics by preparing appropriate superposition states of the HD rotational state. We note that the SARP technology has the capability to achieve such flexible control of molecular states and it is applicable to a broad class of molecules including those with no permanent electric dipole moment $[18,23]$.

These advances now allow controlled studies of molecular collisions and chemical reactions using fully tailored molecular initial rovibrational states $(v, j, m)$ and their coherent superpositions, where $m$ is the projection of $j$ onto a quantization axis. Such investigations of systems beyond $\mathrm{HD}+\mathrm{H}_{2}$, $\mathrm{HD}+\mathrm{D}_{2}$, and $\mathrm{HD}+\mathrm{He}$ are crucial to unveil whether the stereodynamic control is possible for broader classes of molecules with stronger intermolecular interactions. In particular, the existence and possible control of any stereodynamic effect in the presence of multiple (overlapping) resonances in cold collisions remain to be addressed.

In this Rapid Communication, we examine this question by tackling rotational quenching of $\mathrm{HCl}$ by collisions with $\mathrm{H}_{2}$ in the subkelvin regime based on full-dimensional quantum scattering calculations on a recently reported $a b$ initio 
potential energy surface (PES) [24]. This system is of current experimental interest in Zare's group (private communication). In comparison with $\mathrm{HD}$, the $\mathrm{HCl}$ molecule is strongly dipolar (dipole moment $\sim 1.08 \mathrm{D}$ ) and is amenable to high optical controllability of its internal states. The $\mathrm{HCl}+\mathrm{H}_{2}$ interaction potential is deeper and more anisotropic [24] leading to strong coupling between the different rovibrational channels at short range. The small rotational constant of $\mathrm{HCl}$ (a factor of 5 smaller than HD) also contributes to the increased density of states and more complex collision dynamics for the $\mathrm{HCl}+\mathrm{H}_{2}$ system.

Details of the $a b$ initio $\mathrm{PES}$ for $\mathrm{HCl}+\mathrm{H}_{2}$ and its analytical representation have been reported previously [24]. Briefly, the PES consists of a short-range part fit to high-level $a b$ initio data and a long-range part giving an accurate description of the electrostatic and dispersion interactions. The depth of the interaction potential well is $\sim 216 \mathrm{~cm}^{-1}$, significantly deeper than that between two $\mathrm{H}_{2}$ molecules $\left(\sim 35 \mathrm{~cm}^{-1}\right)$. The timeindependent Schrödinger equation for the molecular scattering is numerically solved using the close-coupling method as implemented in the TwoBC code $[25,26]$. More details of the computational approach can be found in previous publications $[20,21,24]$ and we limit our discussions to key notations and essential formulas.

The state-to-state differential cross section (DCS) for isotropic collisions (no polarization) in the helicity representation is given by [27]

$$
\begin{aligned}
\frac{d \sigma_{\alpha \rightarrow \alpha^{\prime}}}{d \Omega}= & \frac{1}{\left(2 j_{1}+1\right)\left(2 j_{2}+1\right)} \\
& \times \sum_{k_{1}, k_{2}, k_{1}^{\prime}, k_{2}^{\prime}}\left|q_{\alpha, k_{1}, k_{2} \rightarrow \alpha^{\prime}, k_{1}^{\prime}, k_{2}^{\prime}}\right|^{2},
\end{aligned}
$$

where $\alpha \equiv v_{1} j_{1} v_{2} j_{2}$ and $\alpha^{\prime} \equiv v_{1}^{\prime} j_{1}^{\prime} v_{2}^{\prime} j_{2}^{\prime}$ refer to the initial and final combined molecular rovibrational states, respectively, $d \Omega$ is the infinitesimal solid angle, and the subscript 1 refers to $\mathrm{HCl}$ and 2 to $\mathrm{H}_{2}$. The quantum number $k / k^{\prime}$ is the projection of the initial/final molecular rotation angular momentum $j / j^{\prime}$ onto the body-fixed (BF) $z$ axis. The $\theta$ dependence in the scattering amplitude is given as $[20,21,27]$

$$
\begin{aligned}
& q_{\alpha, k_{1}, k_{2} \rightarrow \alpha^{\prime}, k_{1}^{\prime}, k_{2}^{\prime}} \frac{1}{2 k_{\alpha}} \sum_{J}(2 J+1) \sum_{j_{12}, j_{12}^{\prime}, l, l^{\prime}} i^{l-l^{\prime}+1} T_{\alpha j_{12} l, \alpha^{\prime} j_{12}^{\prime} l^{\prime}}^{J}(E) d_{k_{12}, k_{12}^{\prime}}^{J}(\theta) \\
& \quad \times\left\langle j_{12}^{\prime} k_{12}^{\prime} J-k_{12}^{\prime} \mid l^{\prime} 0\right\rangle\left\langle j_{12} k_{12} J-k_{12} \mid l 0\right\rangle \\
& \quad \times\left\langle j_{1}^{\prime} k_{1}^{\prime} j_{2}^{\prime} k_{2}^{\prime} \mid j_{12}^{\prime} k_{12}^{\prime}\right\rangle\left\langle j_{1} k_{1} j_{2} k_{2} \mid j_{12} k_{12}\right\rangle,
\end{aligned}
$$

where $k_{\alpha}$ is the wave number for the incident channel, and $J$ is the quantum number of the total angular momentum of the collision complex defined as $\boldsymbol{J}=\boldsymbol{j}_{12}+\boldsymbol{l}$ with the combined rotational angular momentum $\boldsymbol{j}_{12}$ of the two molecules given by $j_{12}=j_{1}+j_{2}$ and the relative orbital angular momentum $l$ between the collision partners. The $T$ matrix is given in terms of the $S$ matrix as $T=1-S, E$ is the total energy, and $d_{k_{12}, k_{12}^{\prime}}^{J}(\theta)$ is the Wigner's reduced rotation matrix, and the braket $\langle\mid\rangle$ denotes a Clebsch-Gordan coefficient. We note that $k_{12}$ and $k_{12}^{\prime}$ in Eq. (2) mean $k_{1}+k_{2}$ and $k_{1}^{\prime}+k_{2}^{\prime}$, respectively.

By taking the integral of the DCS in Eq. (1) over $\theta$ and $\phi$ one obtains the state-to-state integral cross section (ICS) as
$[24,26,27]$

$$
\begin{aligned}
\sigma_{\alpha \rightarrow \alpha^{\prime}}(E)= & \frac{\pi}{\left(2 j_{1}+1\right)\left(2 j_{2}+1\right) k_{\alpha}^{2}} \\
& \times \sum_{j_{12} j_{12}^{\prime}, l l^{\prime} J}(2 J+1)\left|\delta_{\alpha j_{12} l, \alpha^{\prime} j_{12}^{\prime} l^{\prime}}-S_{\alpha j_{12} l, \alpha^{\prime} j_{12}^{\prime} l^{\prime}}^{J}(E)\right|^{2} .
\end{aligned}
$$

Equations (1) and (3) include averaging over all possible initial orientations of the molecular rotational states specified by the projections $k_{1}$ and $k_{2}$. Our primary goal is to explore the effect of aligning the $\mathrm{HCl}$ bond axis relative to the initial relative velocity vector for the collision $(\mathrm{BF} z$ axis), as explored in previous studies of HD [11,12,21,22]. In this scheme the initial rotational state of $\mathrm{HCl}$ is prepared as $\left|j_{1}, \tilde{m}_{1}=0\right\rangle$ by the laboratory fixed SARP laser where $\tilde{m}_{1}$ is the projection of $j_{1}$ onto the linear polarization direction of the SARP laser. The prepared state $\left|j_{1}, \tilde{m}_{1}=0\right\rangle$ can be expressed as a coherent superposition of a set of states $\left|j_{1}, k_{1}\right\rangle$ specified by the projection $j_{1}$ onto the $\mathrm{BF} z$ axis as [21]

$$
\left|j_{1}, \tilde{m}_{1}=0\right\rangle=\sum_{k_{1}=-j_{1}}^{j_{1}} d_{\tilde{m}_{1}=0, k_{1}}^{j_{1}}(\beta)\left|j_{1}, k_{1}\right\rangle,
$$

where $\beta$ is the angle between the aligned $\mathrm{HCl}$ bond axis and the initial relative velocity vector for the collision.

For rotational quenching of aligned $\mathrm{HCl}$ by cold collision with para- $\mathrm{H}_{2}\left(v_{2}=0, j_{2}=0, k_{2}=0\right)$, the state-to-state DCS with respect to the scattering angle $\theta$ is given by [21]

$$
\left(\frac{d \sigma}{d \theta}\right)_{\beta}=2 \pi \sin \theta \sum_{k_{1}=-j_{1}}^{j_{1}} \sum_{k_{1}^{\prime}=-j_{1}^{\prime}}^{j_{1}^{\prime}}\left|d_{0, k_{1}}^{j_{1}}(\beta)\right|^{2}\left|q_{\alpha, k_{1}, 0 \rightarrow \alpha^{\prime}, k_{1}^{\prime}, 0}\right|^{2},
$$

where it is assumed that, like in previous experiments [11-13], the rotationally deexcited $\mathrm{HCl}$ molecules in a specific rotational state $\left(j_{1}^{\prime}\right)$ are detected with no resolution of their $k_{1}^{\prime}$ or azimuthal angle $\phi$. The overall effect of the alignment of the $\mathrm{HCl}$ molecules is captured in the weight factor $\left|d_{0, k_{1}}^{j_{1}}(\beta)\right|^{2}$ for each $|q|^{2}$ term associated with an initial $k_{1}$. The behavior of $\left|d_{0, k_{1}}^{j_{1}}(\beta)\right|^{2}$ as a function of $\beta$ for $j_{1}=2$ is displayed in Ref. [21] (see also Fig. S1 in the Supplemental Material [28]).

First we discuss rotational quenching of $\mathrm{HCl}\left(v_{1}=\right.$ $\left.1, j_{1}=2\right) \rightarrow\left(v_{1}^{\prime}=1, j_{1}^{\prime}=0\right)$ by collisions with para $-\mathrm{H}_{2}$ $\left(v_{2}=0, j_{2}=0, k_{2}=0\right.$ ), namely, $\Delta j_{1}=-2$. The ICS for isotropic collisions is shown by the dashed curve in Fig. 1 . Contributions from different incoming partial waves $l=0$ to $l=3$ are also shown. It is seen that a pronounced peak at about a collision energy of $E_{C}=0.3 \mathrm{~cm}^{-1}$ has the main contribution $(77 \%)$ from an incoming $p$-wave $(l=1)$ and a smaller contribution from an incoming $f$-wave $(l=3)$. The quasibound states due to closed channels are inaccessible at these collision energies (see Fig. S2 in the Supplemental Material [28]), thus the observed resonance is a $p$-wave shape resonance. We note that a $p$-wave shape resonance was not observed for $\mathrm{HD}+$ para- $\mathrm{H}_{2}$ collisions [20-22], indicating that cold $\mathrm{HCl}+\mathrm{H}_{2}$ collisions exhibit markedly different features compared to $\mathrm{HD}+\mathrm{H}_{2}$. Indeed, the adiabatic potential energy curves for $\mathrm{HCl}+\mathrm{H}_{2}$ are much more complicated [28] than those of $\mathrm{HD}+\mathrm{H}_{2}[20]$. 


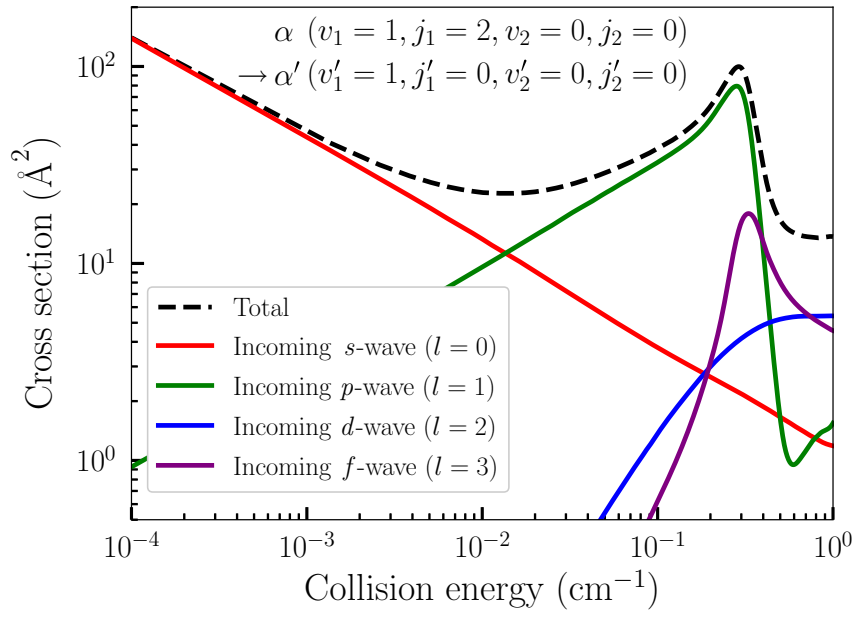

FIG. 1. Integral cross section for the rotational quenching of $\mathrm{HCl}$ $\left(v_{1}=1, j_{1}=2 \rightarrow v_{1}^{\prime}=1, j_{1}^{\prime}=0\right)$ due to collisions with para- $\mathrm{H}_{2}$ $\left(v_{2}=0, j_{2}=0\right)$ for the isotropic case (no alignment). The dashed black curve shows the total cross section and the solid curves display the partial wave contributions.

We now discuss how to control this resonance by the preparation of initial alignment of the $\mathrm{HCl}$ bond axis. The ICS in the resonance region with and without initial alignment of $\mathrm{HCl}$ is shown in Fig. 2(a) using linear axis scale. The dashed curve (isotropic) is identical to the dashed curve (Total) in Fig. 1. Other solid curves correspond to different alignment angles of the $\mathrm{HCl}$ bond axis with respect to the initial relative velocity vector for the collision, obtained by integrating the corresponding DCS over $\theta$ from 0 to $\pi$ in Eq. (5). The cross section varies almost monotonically by a factor of 3 as $\beta$ increases from $0^{\circ}$ to $90^{\circ}$, indicating the feasibility of robust control of quenching rate by changing the angle of alignment.

As shown in Eq. (5), the DCS and the resultant ICS are given as the sum of terms specified by the different projection component $k_{1}$ of $j_{1}$. Therefore, the observed trend of the ICS in terms of $\beta$ can be effectively analyzed by examining the ICS with initially oriented $\mathrm{HCl}$ specified by a projection $k_{1}$ [Fig. 2(b)]. Note that the result for $k_{1}=0$ is the same as $\beta=$ 0 in Fig. 2(a). The cross sections for other values of $k_{1}$ are relatively suppressed in the resonance region. In particular, a shallow dip is observed for $\left|k_{1}\right|=2$.

The above findings indicate that the sensitive dependence of the dynamics in the resonance region to the initial orientation (specified by $k_{1}$ ) of $\mathrm{HCl}$ can cause dramatic change in collision outcome. Compared to $\left|k_{1}\right|=2$, the ICS for $k_{1}=0$ is about 12 -fold larger at the peak of the resonance $\left(E_{C}=0.3 \mathrm{~cm}^{-1}\right)$. Since the projections of the final rotational states are $k_{1}^{\prime}=0$ and $k_{2}^{\prime}=k_{2}=0$ due to $j_{1}^{\prime}=0$ and $j_{2}^{\prime}=$ $j_{2}=0$, collisions with $k_{1}=0$ conserve helicity as $k_{1}+k_{2}=$ $k_{1}^{\prime}+k_{2}^{\prime}=0$ and the resonance enhancement can be attributed to a helicity conserving transition. It is worth pointing out that results in Fig. 2(a) do not reflect the full potential of stereodynamic control. If it is possible to specifically prepare an initial $\mathrm{HCl}$ rotational state with $k_{1}=2$ or $k_{1}=-2$, or their linear combination, the resonance can be completely switched off. Recent advances in SARP techniques have made progress in this direction $[13,19]$. Indeed, preparation of a phase-locked
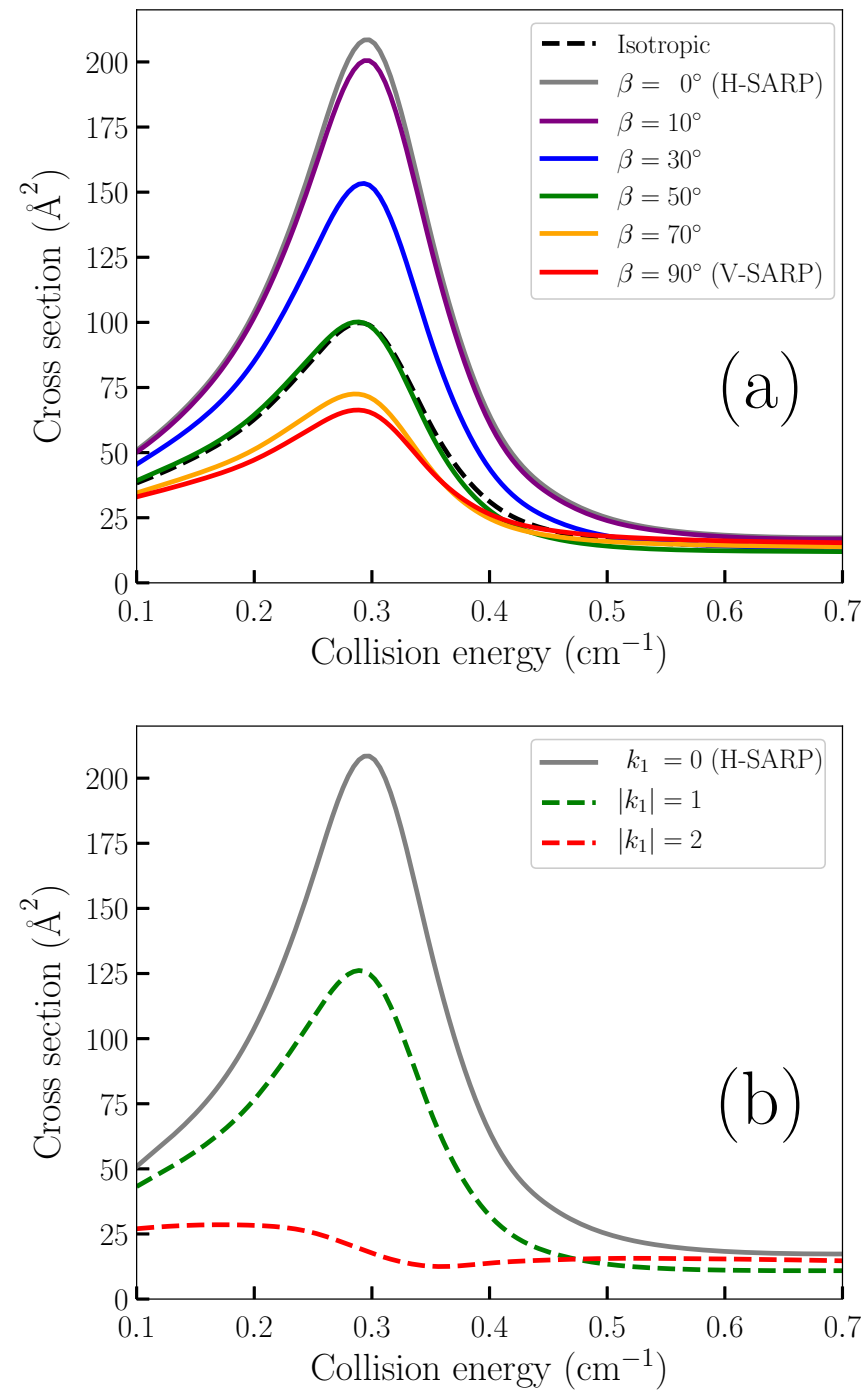

FIG. 2. Integral cross section for $\Delta j=-2$ transition in $\mathrm{HCl}$ : (a) for different alignments of $\mathrm{HCl}$ bond axis against the initial relative velocity vector for the collision; (b) for different orientations specified by the projection $k_{1}$ of the initial rotational state of $\mathrm{HCl}$ $\left(j_{1}=2\right)$. The result for $k_{1}=0$ is identical to that of $\beta=0^{\circ}$ in (a).

superposition of $k_{1}= \pm 1$ states, $\sqrt{1 / 2}\left(\left|j_{1}=2, k_{1}=1\right\rangle+\right.$ $\left.\left|j_{1}=2, k_{1}=-1\right\rangle\right)$, termed X-SARP, was demonstrated for HD [13], expanding the range of control achievable using SARP techniques. The range of control expands into the nonresonant region for the DCS as shown in the Supplemental Material [28].

Next we examine $\Delta j_{1}=-1$ transition in $\mathrm{HCl}$, i.e., $\left(v_{1}=\right.$ $\left.1, j_{1}=2\right) \rightarrow\left(v_{1}^{\prime}=1, j_{1}^{\prime}=1\right)$. Figure 3 presents the total and partial wave resolved ICSs for this process for the isotropic case. The crucial difference here in comparison with Fig. 1 is the enhanced incoming $f$-wave contribution at around $0.4 \mathrm{~cm}^{-1}$ in addition to a strong $p$-wave resonance at about $0.15 \mathrm{~cm}^{-1}$ leading to two prominent peaks. While the two resonances appear as distinct peaks in close proximity we note that the $p$-wave peak has a small contribution from the $f$-wave resonance illustrating the complex nature of the dynamics and partial overlap of these resonances. This brings up an important question that has not been addressed before: Can 


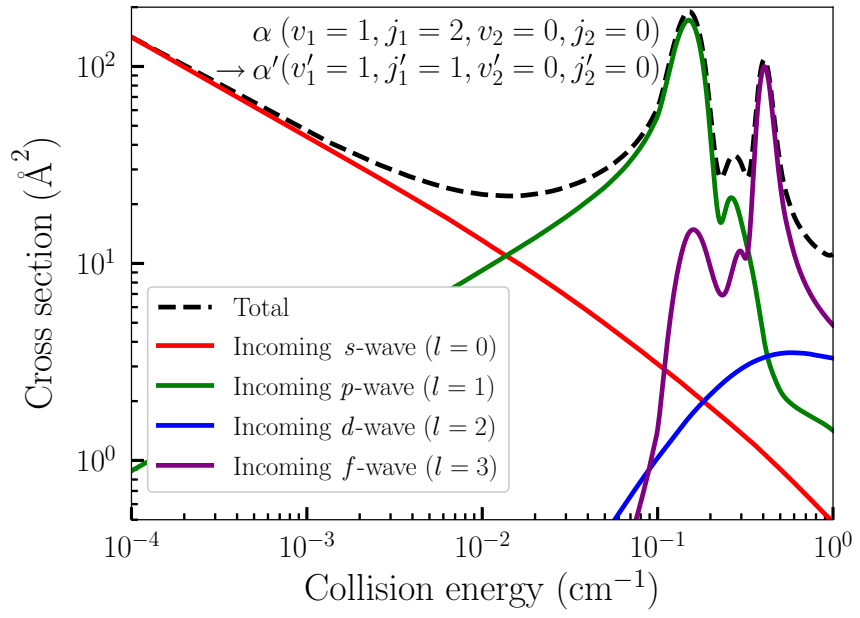

FIG. 3. The same as in Fig. 1 but for $\Delta j=-1$ transition.

stereodynamic control be effective for such overlapping resonances associated with multiple partial waves? In previous studies of $\mathrm{HD}+\mathrm{H}_{2}, \mathrm{HD}+\mathrm{D}_{2}$, and $\mathrm{HD}+\mathrm{He}$, the stereodynamic effect was attributed to the characteristics of a specific isolated shape resonance. However, such simplicity is not necessarily expected for the vast majority of systems due to the higher density of states caused by the stronger interaction and/or heavier mass.

As shown in Fig. 4(a) the angle ( $\beta$ ) between the aligned $\mathrm{HCl}$ bond axis and the initial relative velocity vector for the collision has a strong effect on the magnitude of both resonances. However, some notable differences are also visible compared to the $\Delta j_{1}=-2$ transition [Fig. 2(a)]. In Fig. 4(a), the $f$-wave shape resonance peak increases in intensity with increasing $\beta$ from $0^{\circ}$ to $90^{\circ}$, just the opposite of the trend observed for the peak in the $\Delta j_{1}=-2$ transition. The $p$-wave peak at lower energy increases in intensity with increasing $\beta$ but it saturates at around $50^{\circ}$ before eventually decreasing with increasing $\beta$ (from $70^{\circ}$ to $90^{\circ}$ ). Nevertheless, both resonances exhibit a considerable range of control by the alignment preparation. In particular, with a small value of $\beta$ $\left(<10^{\circ}\right)$, both peaks are essentially switched off.

To gain more insights into the two resonances Fig. 4(b) presents the ICS with different initial $\mathrm{HCl}$ orientations. The cross section for $k_{1}=0$ is the smallest near the resonance peaks and exhibits a small bump near $0.3 \mathrm{~cm}^{-1}$, the region in between the two resonances. On the other hand, we observe two intense peaks with $\left|k_{1}\right|=1$ and 2 , revealing the origin of the overlapping resonances. Unlike the $\Delta j_{1}=-2$ results shown in Fig. 2(b), $\left|k_{1}\right|=2$ gives the largest cross sections while it does not conserve the helicity $\left(k_{1}+k_{2} \neq k_{1}^{\prime}+k_{2}^{\prime}\right)$. It means that the resonances for $\Delta j_{1}=-2$ and $\Delta j_{2}=-1$ transitions do not share the same mechanism. While the interaction potential does not directly contribute to a change in the helicity $k_{1}+k_{2}$, the centrifugal term can induce it (Coriolis coupling), and the combined effect of these terms determines the resulting resonance profile. Recent studies of molecular reaction and spin-relaxation in the cold energy regime have also pointed out that approximate calculations assuming the conservation of helicity tend to fail in resonance regions $[29,30]$.
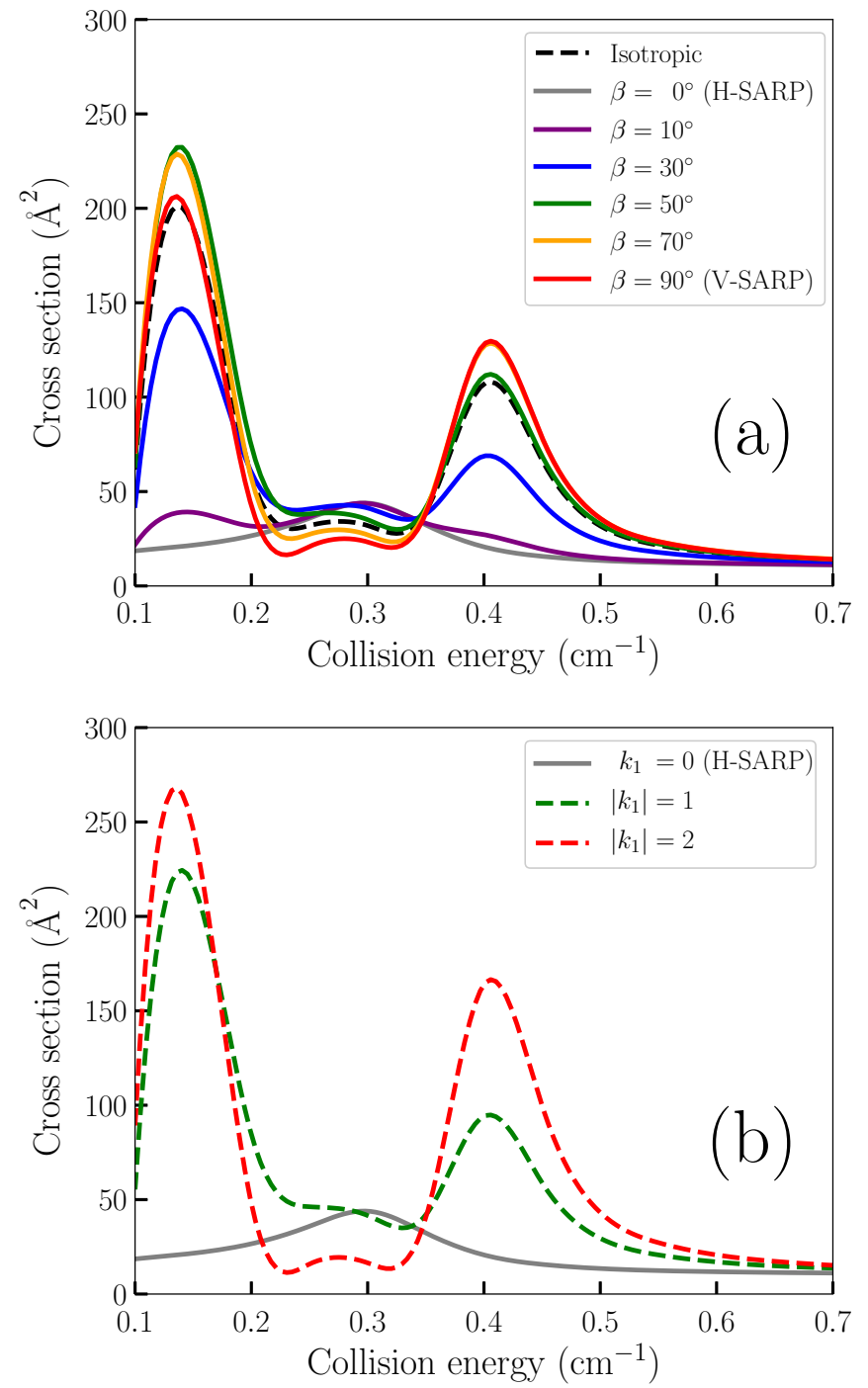

FIG. 4. The same as in Fig. 2 but for $\Delta j=-1$ transition.

In summary, we have demonstrated flexible and robust control of resonant rotational quenching of $\mathrm{HCl}$ in cold collisions with $\mathrm{H}_{2}$ at the level of integral cross sections and rates by preparing $\mathrm{HCl}$ in an aligned or oriented state in the $v=1$ vibrational level. The most striking result of our study is that even multiple peaks due to resonances associated with disparate partial waves can be controlled, indicating that stereodynamic control is not limited to simple systems with isolated resonances. Our results also reveal the importance of helicity-nonconserving transitions in stereodynamic control of resonant molecular collisions. The analysis based on the initial orientations of the $\mathrm{HCl}$ rotational state demonstrates the enormous potential controllability in cold molecular collisions, which is tractable with current experimental techniques.

We are grateful to James Croft and Nandini Mukherjee for stimulating discussions. This work is supported in part by NSF Grant No. PHY-1806334 (N.B.) and ARO MURI Grant No. W911NF-19-1-0283 (N.B. and H.G.). 
[1] K. M. Jones, E. Tiesinga, P. D. Lett, and P. S. Julienne, Ultracold photoassociation spectroscopy: Long-range molecules and atomic scattering, Rev. Mod. Phys. 78, 483 (2006).

[2] R. V. Krems, Cold controlled chemistry, Phys. Chem. Chem. Phys. 10, 4079 (2008).

[3] L. D. Carr, D. DeMille, R. V. Krems, and J. Ye, Cold and ultracold molecules: Science, technology and applications, New J. Phys. 11, 055049 (2009).

[4] C. Naulin and M. Costes, Experimental search for scattering resonances in near cold molecular collisions, Int. Rev. Phys. Chem. 33, 427 (2014).

[5] B. K. Stuhl, M. T. Hummon, and J. Ye, Cold state-selected molecular collisions and reactions, Annu. Rev. Phys. Chem. 65, 501 (2014).

[6] N. Balakrishnan, Perspective: Ultracold molecules and the dawn of cold controlled chemistry, J. Chem. Phys. 145, 150901 (2016).

[7] J. L. Bohn, A. M. Rey, and J. Ye, Cold molecules: Progress in quantum engineering of chemistry and quantum matter, Science 357, 1002 (2017).

[8] M. S. Safronova, D. Budker, D. DeMille, Derek F. Jackson Kimball, A. Derevianko, and C. W. Clark, Search for new physics with atoms and molecules, Rev. Mod. Phys. 90, 025008 (2018).

[9] Y. Shagam, A. Klein, W. Skomorowski, R. Yun, V. Averbukh, C. P. Koch, and E. Narevicius, Molecular hydrogen interacts more strongly when rotationally excited at low temperatures leading to faster reactions, Nat. Chem. 7, 921 (2015).

[10] Y. Segev, M. Pitzer, M. Karpov, N. Akerman, J. Narevicius, and E. Narevicius, Collisions between cold molecules in a superconducting magnetic trap, Nature (London) 572, 189 (2019).

[11] W. E. Perreault, N. Mukherjee, and R. N. Zare, Quantum control of molecular collisions at 1 kelvin, Science 358, 356 (2017).

[12] W. E. Perreault, N. Mukherjee, and R. N. Zare, Cold quantumcontrolled rotationally inelastic scattering of $\mathrm{HD}$ with $\mathrm{H}_{2}$ and $\mathrm{D}_{2}$ reveals collisional partner reorientation, Nat. Chem. 10, 561 (2018).

[13] W. E. Perreault, N. Mukherjee, and R. N. Zare, HD ( $v=1$, $j=2, m)$ orientation controls HD-He rotationally inelastic scattering near $1 \mathrm{~K}, \mathrm{~J}$. Chem. Phys. 150, 174301 (2019).

[14] R. N. Zare, Laser control of chemical reactions, Science 279, 1875 (1998).

[15] A. J. Orr-Ewing, Dynamical stereochemistry of bimolecular reactions, J. Chem. Soc., Faraday Trans. 92, 881 (1996).

[16] J. Aldegunde, M. P. de Miranda, J. M. Haigh, B. K. Kendrick, V. Sáez-Rábanos, and F. J. Aoiz, How reactants polarization can be used to change and unravel chemical reactivity, J. Phys. Chem. A 109, 6200 (2005).
[17] F. Wang, K. Liu, and T. P. Rakitzis, Revealing the stereospecific chemistry of the reaction of $\mathrm{Cl}$ with aligned $\mathrm{CHD}_{3}\left(v_{1}=1\right)$, Nat. Chem. 4, 636 (2012).

[18] W. Dong, N. Mukherjee, and R. N. Zare, Optical preparation of $\mathrm{H}_{2}$ rovibrational levels with almost complete population transfer, J. Chem. Phys. 139, 074204 (2013).

[19] N. Mukherjee, W. Dong, and R. N. Zare, Coherent superposition of M-states in a single rovibrational level of $\mathrm{H}_{2}$ by Starkinduced adiabatic Raman passage, J. Chem. Phys. 140, 074201 (2014).

[20] J. F. E. Croft, N. Balakrishnan, M. Huang, and H. Guo, Unraveling the Stereodynamics of Cold Controlled HD- $\mathrm{H}_{2}$ Collisions, Phys. Rev. Lett. 121, 113401 (2018).

[21] J. F. E. Croft and N. Balakrishnan, Controlling rotational quenching rates in cold molecular collisions, J. Chem. Phys. 150, 164302 (2019).

[22] P. G. Jambrina, J. F. E. Croft, H. Guo, M. Brouard, N. Balakrishnan, and F. J. Aoiz, Stereodynamical Control of a Quantum Scattering Resonance in Cold Molecular Collisions, Phys. Rev. Lett. 123, 043401 (2019).

[23] W. E. Perreault, N. Mukherjee, and R. N. Zare, Stark-induced adiabatic Raman passage examined through the preparation of $\mathrm{D}_{2}(v=2, j=0)$ and $\mathrm{D}_{2}(v=2, j=2, m=0), \mathrm{J}$. Chem. Phys. 150, 234201 (2019).

[24] Q. Yao, M. Morita, C. Xie, N. Balakrishnan, and H. Guo, Globally accurate full-dimensional potential energy surface for $\mathrm{H}_{2}+\mathrm{HCl}$ inelastic scattering, J. Phys. Chem. A 123, 6578 (2019).

[25] R. V. Krems, TwoBC Quantum Scattering Program (University of British Columbia, Vancouver, 2006).

[26] G. Quéméner and N. Balakrishnan, Quantum calculations of $\mathrm{H}_{2}-\mathrm{H}_{2}$ collisions: From ultracold to thermal energies, J. Chem. Phys. 130, 114303 (2009).

[27] J. Schaefer and W. Meyer, Theoretical studies of $\mathrm{H}_{2}-\mathrm{H}_{2}$ collisions. I. Elastic scattering of ground state para and ortho$\mathrm{H}_{2}$ in the rigid rotor approximation, J. Chem. Phys. 70, 344 (1979).

[28] See Supplemental Material at http://link.aps.org/supplemental/ 10.1103/PhysRevResearch.2.032018 for squared Wigner's reduced rotational matrix elements, adiabatic and diabatic potential energy curves, differential cross section, and integral cross section for minor transitions associated with vibrational relaxation of $\mathrm{HCl}$.

[29] G. Quéméner and N. Balakrishnan, Cold and ultracold chemical reactions of $\mathrm{F}+\mathrm{HCl}$ and $\mathrm{F}+\mathrm{DCl}, \mathrm{J}$. Chem. Phys. 128, 224304 (2008).

[30] M. Morita and T. V. Tscherbul, Restricted basis set coupledchannel calculations on atom-molecule collisions in magnetic fields, J. Chem. Phys. 150, 074110 (2019). 